\title{
Alterações da Função Pulmonar após Tratamento Cirúrgico de Cardiopatias Congênitas com Hiperfluxo Pulmonar
}

\author{
Changes in Pulmonary Function after Surgical Treatment of Congenital Heart Disease with Pulmonary Hyperflow \\ Lilian Goraieb ${ }^{1}$, Ulisses Alexandre Croti ${ }^{1}$, Suzana Renata Perez Orrico ${ }^{1}$, Omar Yesid Prieto Rincon², Domingo \\ Marcolino Braile \\ Hospital de Base da Fundação Regional de Medicina (FUNFARME / FAMERP), São José do Rio Preto, São Paulo', Instituto do Coração (INCOR), \\ Brasilia, $D F^{2}-$ Brasil
}

\section{Resumo}

Fundamento: Análise das condições pulmonares dos pacientes no pós-operatório de cirurgia cardíaca pediátrica.

Objetivo: Avaliar o comportamento da complacência pulmonar e resistência da via aérea nos pacientes portadores de cardiopatias congênitas com hiperfluxo pulmonar, submetidos a tratamento cirúrgico com auxílio de circulação extracorpórea.

Métodos: Avaliaram-se, durante a cirurgia, 35 pacientes com medidas de complacência estática e resistência da via aérea, em quatro instantes distintos. As medidas pulmonares foram feitas de forma não-invasiva, com o método de oclusão da via aérea ao final da inspiração e uso de fórmulas matemáticas específicas. As variáveis observadas e relacionadas às alterações pulmonares foram: no período pré-operatório, idade, peso e relação entre fluxo sangüíneo sistêmico e pulmonar; no intra-operatório, tempos de perfusão, de anóxia e temperatura mínima; no pós-operatório, tempo de ventilação mecânica e de permanência na unidade de terapia intensiva.

Resultados: Ao final da cirurgia, a complacência pulmonar mostrou aumento significativo imediato (p $<0,001)$ em todos os pacientes. Os pacientes maiores de 30 meses tiveram maior aumento $(p=0,0004)$. Aqueles com peso superior a $10 \mathrm{~kg}$ também apresentaram maior aumento $(p=0,0006)$. Os pacientes com tempo de circulação extracorpórea maior que 50 minutos demoraram mais para apresentar aumento da complacência pulmonar $(p=0,04)$. A resistência da via aérea não apresentou alteração significativa ao final da correção cirúrgica $(p=0,393)$.

Conclusão: A complacência pulmonar apresentou melhora ao final da cirurgia em todos os pacientes, sendo influenciada de forma significativa por fatores como idade, peso e tempo de circulação extracorpórea. A resistência da via aérea, entretanto, não se alterou. (Arq Bras Cardiol 2008;91(2):77-84)

Palavras-chave: Cardiopatias congênitas, complacência pulmonar, circulação extracorpórea.

\section{Summary}

Background: Analysis of pulmonary status of pediatric patients in the postoperative phase of cardiac surgery.

Objective: To assess pulmonary compliance and airway resistance in patients with congenital heart disease and pulmonary hyperflow submitted to surgical treatment with the use of extracorporeal circulation.

Methods: Thirty-five patients were evaluated during surgery with measurements of static compliance and airway resistance at four different timepoints. Pulmonary measurements were performed non-invasively using end-inspiratory airway occlusion and specific mathematical formulas. The variables examined and related to pulmonary changes were: preoperative - age, weight, and relationship between systemic and pulmonary blood flow; intraoperative - perfusion times, anoxia times and minimum temperature; postoperative - time on mechanical ventilation and length of stay in the intensive care unit.

Results: Pulmonary compliance in all patients had an immediate and significant increase $(P<0.001)$ at the end of surgery. Patients older than 30 months experienced a greater increase $(P=0.0004)$. Those with more than $10 \mathrm{~kg}$ also had a greater increase $(P=0.0006)$. In patients on extracorporeal circulation for more than 50 minutes, the increase in pulmonary compliance took longer to occur $(P=0.04)$. Airway resistance was not significantly changed at the end of surgery $(P=0.393)$.

Conclusion: All patients experienced improved pulmonary compliance at the end of surgery, and this was significantly influenced by age, weight and time on extracorporeal circulation. Airway resistance, however, was not changed. (Arq Bras Cardiol 2008;91(2):70-76)

Key words: Heart defects, congenital; lung compliance; extracorporeal circulation.

Full texts in English - http://www.arquivosonline.com.br

Correspondência: Lilian Goraieb •

Rua Duarte Pacheco, 1401/Casa 32, Higienópolis, 15.085-140, São José do Rio Preto, SP - Brasil

E-mail: liliangoraieb@yahoo.com.br

Artigo recebido em 01/11/07; revisado recebido em 04/12/07; aceito em 26/12/07. 


\section{Introdução}

O hiperfluxo pulmonar nos portadores de cardiopatias congênitas pode provocar mudança na mecânica pulmonar, aumentando o trabalho respiratório e o consumo de oxigênio, com agravo da insuficiência cardíaca ${ }^{1}$.

A função pulmonar pode se encontrar comprometida, com alterações da complacência pulmonar $(\mathrm{Cp})$ e resistência da via aérea $\left(R_{V A}\right)^{2}$.

A correção do defeito com conseqüente normalização do fluxo deve ser realizada em momento adequado para cada paciente, na dependência do grau de repercussão clínica e magnitude do defeito.

Comumente, a via de acesso utilizada no tratamento cirúrgico é a toracotomia transesternal mediana, a qual altera a anatomia torácica durante o procedimento e pode interferir na função pulmonar ${ }^{3}$.

A necessidade do auxílio da circulação extracorpórea (CEC), quando por determinado período os pulmões não são ventilados e permanecem colapsados, propicia o edema pulmonar com conseqüente diminuição da Cp e aumento da $R_{\mathrm{VA}}{ }^{3}$.

Assim, procuramos avaliar as alterações da função pulmonar, por meio das medidas de complacência pulmonar e resistência da via aérea, nos pacientes portadores de cardiopatias congênitas com hiperfluxo pulmonar, submetidos a tratamento cirúrgico com auxílio de circulação extracorpórea.

\section{Métodos}

O estudo foi realizado no Hospital de Base da Fundação Faculdade Regional de Medicina (Funfarme) de São José do Rio Preto, São Paulo.

No período de fevereiro de 2004 a fevereiro de 2005, foram observados, prospectivamente, 35 pacientes portadores de cardiopatia congênita com hiperfluxo pulmonar, submetidos à correção cirúrgica com auxílio de CEC. Treze $(37,1 \%)$ eram do sexo masculino e $22(62,9 \%)$ do sexo feminino. A idade variou de 4 a 115 meses, com média de 59,23 \pm 43,01 meses e mediana de 63 meses. O peso variou de $3,9 \mathrm{~kg}$ a $36,2 \mathrm{~kg}$, com média de 17,45 $\pm 10,38 \mathrm{~kg}$ e mediana de $17 \mathrm{~kg}$. A relação entre o fluxo pulmonar e sistêmico (Qp/Qs) variou de 1 a 7 , com média de 2,92 $\pm 1,29$.

Foram analisadas variáveis pré-operatórias, intraoperatórias e pós-operatórias.

O estudo foi aprovado pelo Comitê de Ética em Pesquisa da Faculdade de Medicina de São José do Rio Preto, São Paulo, pelo Parecer no 012/2004, em 16 de fevereiro de 2004.

Foram incluídos pacientes com diagnóstico de cardiopatia congênita com hiperfluxo pulmonar, portadores de comunicação interventricular (CIV), comunicação interatrial (CIA) e defeito do septo atrioventricular (DSAV) nas formas parcial e total, com idade inferior a 10 anos e necessariamente sob a utilização do auxílio da CEC.

Utilizou-se ecocardiograma até trinta dias antes da operação, para confirmação do diagnóstico e cálculo do Qp/Qs.

Foram sistematicamente excluídos os portadores de outras cardiopatias congênitas ou adquiridas, nascidos prematuros, doenças pulmonares crônicas prévias, síndromes genéticas, operações cardiovasculares ou pulmonares prévias e reações alérgicas na indução anestésica.

A técnica anestésica e ventilatória foi semelhante para todos os pacientes, empregando-se anestesia geral combinada e balanceada associada à raquianestesia.

A medicação pré-anestésica endovenosa foi midazolan 0,1 $\mathrm{mg} / \mathrm{kg}$, seguida de indução inalatória, com máscara facial de sevofluorano a $5 \%$ e $\mathrm{O}_{2}$ a $95 \%$. Após perda de consciência, administraram-se propofol, atracúrio e isofluorano, conforme a necessidade do paciente.

Utilizou-se a ventilação manual com máscara, seguida de intubação traqueal com tubo de diâmetro adequado. Aplicouse a ventilação mecânica (VM) controlada com volume corrente de $10 \mathrm{ml} / \mathrm{kg}$, freqüência respiratória adequada para idade, mantendo a relação de tempo inspiratório e expiratório de 1/1 com fração inspirada de oxigênio $\left(\mathrm{FiO}_{2}\right)$ de 0,6 e pressão expiratória final positiva (PEEP) de zero.

Na raquianestesia, foram utilizadas a bupivacaína isobárica $0,5 \mathrm{mg} / \mathrm{kg}$ e sufentanila $1 \mathrm{mcg} / \mathrm{kg}$.

Todas as crianças receberam dobutamina e/ou milrinone depois da correção cirúrgica, imediatamente após a saída da CEC.

De acordo com protocolo específico, foram coletados dados em três períodos diferentes: pré, intra e pós-operatório.

Previamente à operação, obtiveram-se as seguintes variáveis: idade, sexo, peso e dados ecocardiográficos, como diagnóstico e Qp/Qs.

Durante o procedimento cirúrgico, foram obtidas as seguintes variáveis: tempo de perfusão, tempo de isquemia miocárdica, temperatura mínima, além da verificação dos dados ventilatórios necessários para o cálculo da $C p$ e $R_{v A}$.

O aparelho de VM utilizado foi o Datex Ohmeda Aestiva $3.000^{\circledR}$. Os parâmetros foram ajustados para ventilação controlada a volume e com volume corrente de $10 \mathrm{ml} / \mathrm{kgm}$, fluxo de onda quadrada e freqüência respiratória de acordo com a idade.

Utilizou-se o método de oclusão da via aérea ao final da inspiração, com pausa inspiratória, na qual a pressão traqueal diminui até atingir um platô e fluxo constantes iguais a zero.

Os dados ventilatórios de pressão inspiratória máxima, pressão de platô, volume corrente, $\mathrm{FiO}_{2}$ e PEEP foram obtidos no painel do ventilador. Para a verificação da pressão de platô, foi necessário o ajuste do ventilador. Com o aparelho programado para o modo volume, fez-se configuração pelo menu principal para ajuste da pausa inspiratória. Com o objetivo de manter a relação de tempo inspiratório com tempo expiratório de 1:3, ajustou-se a pausa inspiratória de 25\% do tempo inspiratório. Dessa forma, quando retornamos à tela inicial, a pressão de platô aparece no monitor, a cada ciclo respiratório, logo abaixo da pressão máxima.

Tais dados ventilatórios foram observados e medidos em quatro instantes diferentes e bem definidos:

- A primeira medida, com a criança sedada, curarizada, intubada e sob VM, após o posicionamento dos campos estéreis. 


\section{Artigo Original}

- A segunda, após a esternotomia, pericardiotomia e colocação dos afastadores.

- A terceira, com cinco minutos depois do término da CEC com os afastadores ainda posicionados.

- E a quarta, após o fechamento completo do tórax.

Todos os pacientes foram submetidos à CEC com oxigenador de membrana (Braile Biomédica ${ }^{\circledR}$, Brasil) e bomba de rolete. Ao final da intervenção cirúrgica, os pacientes foram transferidos sob ventilação manual para a unidade de terapia intensiva (UTI) e, posteriormente, instalou-se a VM. Alguns pacientes foram extubados no centro cirúrgico, o que dependeu da gravidade da doença, das características da CEC empregada e da avaliação do anestesista.

As variáveis do pós-operatório observadas na UTI foram: tempo de VM e de permanência na UTI.
Para o cálculo da Cp e $\mathrm{R}_{\mathrm{VA}^{\prime}}$ considerou-se o valor da Cst que foi calculado pela fórmula da figura $1^{4,5}$.

A $R_{V A}$ expressa em centímetros de água $\left(\mathrm{cm} / \mathrm{H}_{2} \mathrm{O}\right)$ foi calculada pela fórmula da figura $2^{4,5}$.

Para a análise estatística de dados, os valores da Cst e de $\mathrm{R}_{\mathrm{VA}}$ foram transformados, respectivamente, em índice de complacência (IC) e índice de resistência (IR). Os índices foram encontrados dividindo-se os valores de complacência e resistência pela superfície corpórea, o que permitiu comparar indivíduos diferentes. Esses índices foram relacionados às variáveis do pré, intra e pós-operatório.

\section{Resultados}

A tabela 1 apresenta cálculos associados ao IC nos quatro instantes de medida e mostra que houve pequena alteração

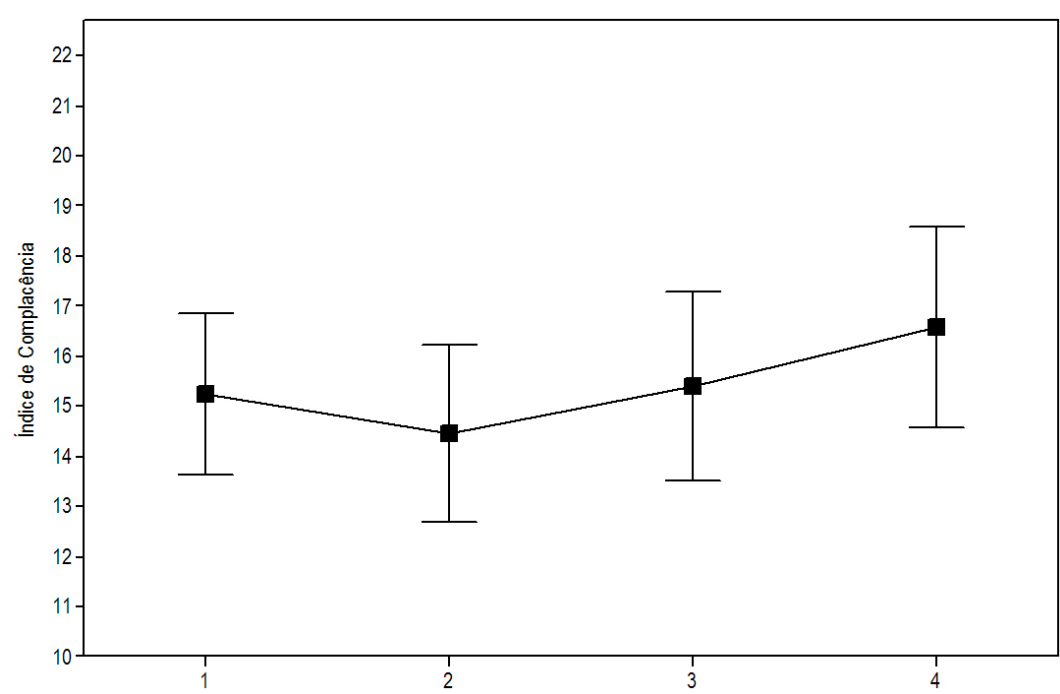

Fig. 1 - Variação do índice de complacência nos quatro instantes.

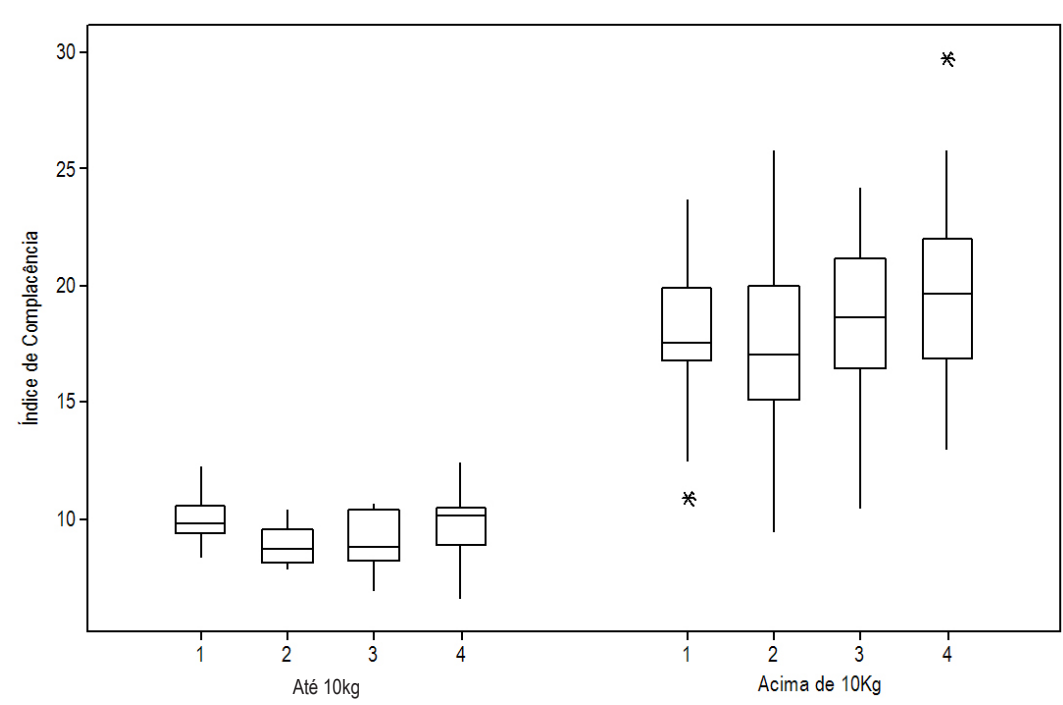

Fig. 2 - Box-plot para dados de índice de complacência, segundo subgrupos de peso. 
Tabela 1 - Variações do IC nos quatro instantes de medida

\begin{tabular}{|c|c|c|c|c|c|c|c|}
\hline IC & $\mathrm{n}$ & Média & DP & Mediana & Extremos & $\begin{array}{c}\text { Quartis } \\
(25 \%-75 \%)\end{array}$ & $\begin{array}{c}\text { Comparações } \\
\text { múltiplas" }^{*}\end{array}$ \\
\hline Instante 1 & 35 & 15,25 & 4,7 & 16,8 & $8,4-23,7$ & $10,4-18,5$ & A \\
\hline Instante 2 & 35 & 14,45 & 5,11 & 15,1 & $7,9-25,8$ & $9,4-18,9$ & A \\
\hline Instante 3 & 35 & 15,40 & 5,52 & 16,5 & $7,0-24,2$ & $10,2-20,3$ & A \\
\hline Instante 4 & 35 & 16,59 & 5,86 & 16,9 & $6,6-29,8$ & $10,3-20,4$ & B \\
\hline
\end{tabular}

IC - indice de complacência; DP - desvio padrão; $n$ - número de pacientes; " letras distintas indicam instantes cujas médias do IC foram estatisticamente diferentes.

nos valores médios do IC, ao passar de um instante para outro, durante o período intra-operatório. Os valores de IC são menos dispersos no instante 1 e atingem a maior variação no instante 4, tendo seu valor médio mais baixo no instante 2. Quando se aplicou o teste de comparação de médias com dois fatores (Two-way ANOVA), verificou-se que as diferenças encontradas foram significativas ( $p<0,001$ ).

As variáveis quantitativas exploradas na análise univariada da relação do índice de complacência foram idade, peso, Qp/Qs, tempo de perfusão, tempo de anóxia, temperatura mínima e tempo de VM e de permanência na UTI.

As variáveis que se mostraram mais fortemente associadas com os valores de IC nos diferentes instantes foram idade, peso e tempo de perfusão. As demais variáveis não apresentaram nenhum padrão de relacionamento.

A tabela 2 apresenta os resultados obtidos para o teste de Mann-Whitney nas comparações de duas amostras estratificadas por idade, nos quatro instantes de medida de IC.

Os resultados referentes ao peso encontram-se na figura 2, com seus intervalos de variação.

Na figura 3 observamos que, relacionando duas variáveis, peso e idade, a maioria dos pacientes com até 30 meses apresenta peso corporal até $10 \mathrm{~kg}$.

Quanto à relação de IC com o tempo de perfusão, a estratificação da amostra, segundo a variável categórica "Tperf_ 50", indicou que há diferença estatisticamente significativa entre os valores medianos em cada instante de avaliação de IC, conforme gráfico Box-plot apresentado na figura 4.

A figura 5 mostra a variação do índice de complacência em

Tabela 2 - Resultados do teste de Mann-Whitney para comparação entre amostras independentes

\begin{tabular}{lcccc}
\hline \multirow{2}{*}{ IC } & \multicolumn{2}{c}{ Idade $\leq \mathbf{3 0}$ meses } & \multicolumn{2}{c}{ Idade $>\mathbf{3 0}$ meses } \\
& $\mathbf{n}$ & Mediana & $\mathbf{n}$ & Mediana \\
\hline Instante 1 & 13 & 9,8 & 22 & 17,7 \\
\hline Instante 2 & 13 & 8,8 & 22 & 17,45 \\
\hline Instante 3 & 13 & 8,8 & 22 & 18,95 \\
\hline Instante 4 & 13 & 10,2 & 22 & 19,85 \\
\hline Valor $p$ & & 0,0165 & & 0,0004 \\
\hline
\end{tabular}

IC - índice de complacência; $n$ - número de pacientes. porcentagem, de acordo com o tempo de perfusão.

A tabela 3 apresenta os cálculos associados ao IR, nos quatro instantes de medida.

\section{Discussão}

As alterações pulmonares no pós-operatório de cirurgia cardíaca pediátrica com CEC são muitas vezes responsáveis pelo aumento da morbidade e mortalidade ${ }^{3,6}$.

Nas crianças portadoras de hiperfluxo pulmonar, observamos alterações na mecânica pulmonar, no pósoperatório com auxílio de CEC.

A média de complacência no instante 4 difere estatisticamente da média nos outros instantes de medição. Isto é, o IC apresentou um aumento estatisticamente significativo ao final da operação.

Doenças que produzem fluxo anormal de sangue nos pulmões podem provocar mudanças na mecânica pulmonar, aumentando o trabalho respiratório e o consumo de oxigênio, e agravando a deficiência cardíaca ${ }^{1}$. A melhora da complacência pulmonar ao final da operação decorre provavelmente da correção do hiperfluxo pulmonar com melhora imediata da mecânica pulmonar. Essa melhora foi traduzida pelo aumento do IC, isto é, quanto maior, mais distensível está o pulmão e com maior variação de volume durante a inspiração; logo quanto menor, mais rígido e menor o volume ${ }^{7}$.

As alterações produzidas por CEC, hipotermia, ausência de ventilação durante a operação e pelo procedimento cirúrgico seriam suficientes para comprometer a função pulmonar no

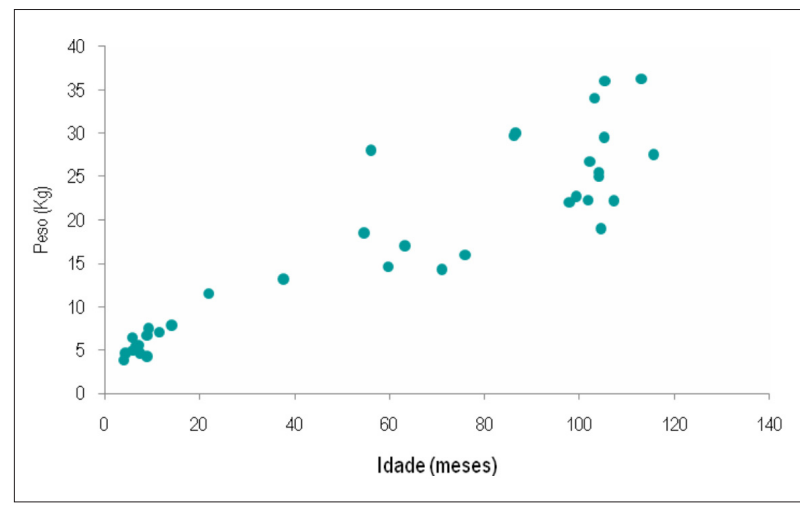

Fig. 3 - Relação entre a idade e o peso. 


\section{Artigo Original}

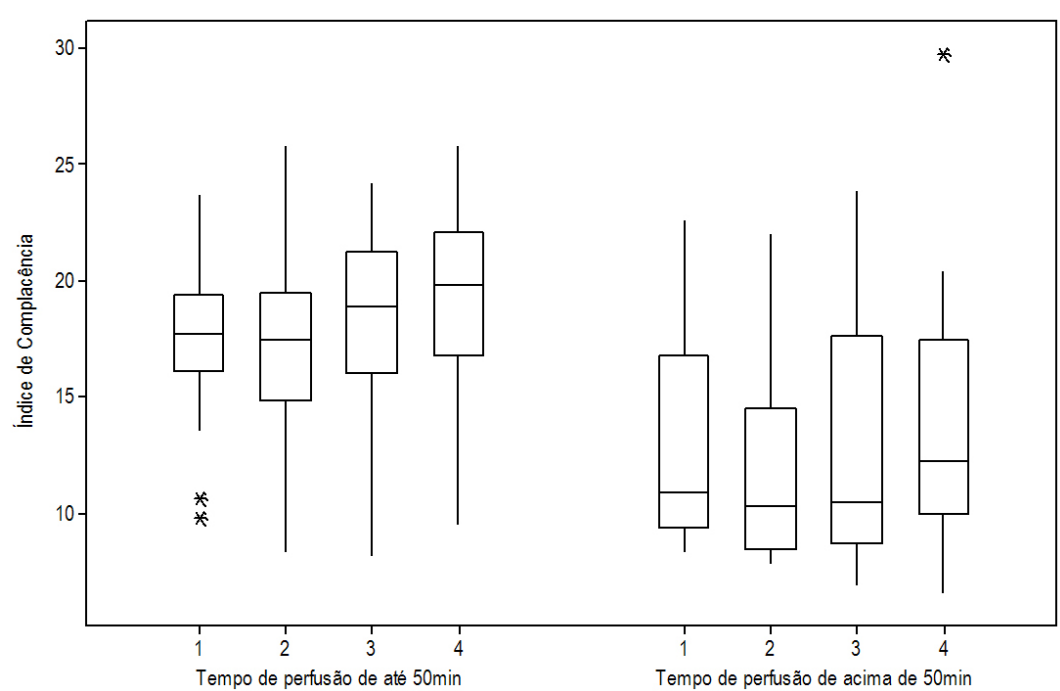

Fig. 4 - Box-plot de índice de complacência, de acordo com subgrupo de tempo de perfusão.

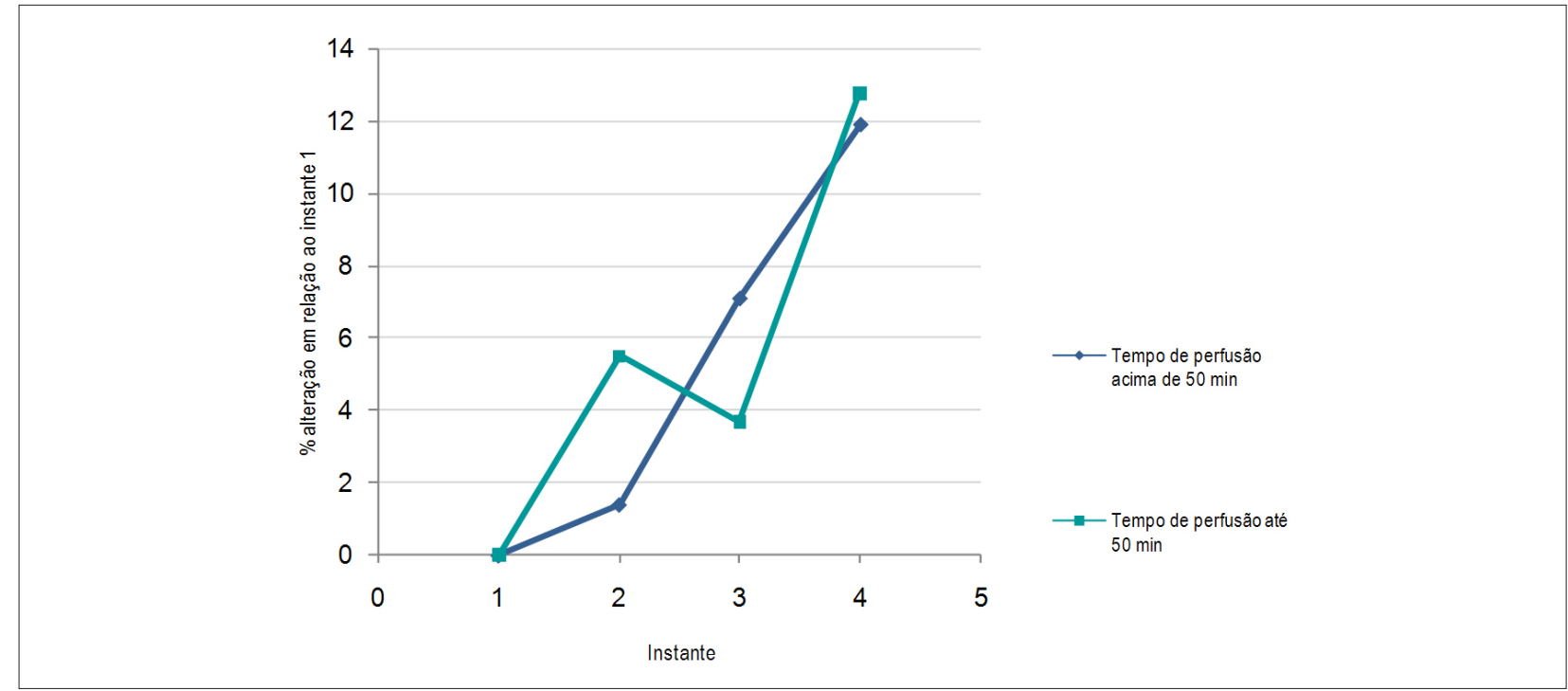

Fig. 5 - Variação em porcentagem do índice de complacência, em relação ao tempo de perfusão.

Tabela 3 - Estatísticas descritivas para índice de resistência por instante do período intra-operatório

\begin{tabular}{lcccccc}
\hline IR & $\mathbf{n}$ & Média & DP & Mediana & Extremos & Quartis $(25 \%-75 \%)$ \\
\hline Instante 1 & 35 & 72,5 & 73,0 & 44,6 & $12,6-332,4$ & $25,8-89,9$ \\
\hline Instante 2 & 35 & 72,7 & 73,9 & 44,4 & $9,1-332,4$ & $26,3-82,9$ \\
\hline Instante 3 & 35 & 75,7 & 80,7 & 53,3 & $12,7-415,5$ & $28,0-91,4$ \\
\hline Instante 4 & 35 & 73,0 & 69,1 & 53,3 & $14,2-282,6$ & $28,7-81,2$ \\
\hline Valor p & & & $p=0,393$ & & & \\
\hline
\end{tabular}

$\mathbb{I R}$ - índice de resistência; $n$ - número de pacientes; DP - desvio padrão.

pós-operatório. Muitas vezes, essas alterações são responsáveis pela morbidade dos pacientes ${ }^{8}$.
Não ocorreu alteração que comprometesse a função pulmonar no pós-operatório, o que foi traduzido pelo aumento 
do IC, comprovando, dessa forma, que os benefícios da correção do hiperfluxo superam possíveis complicações pulmonares decorrentes da CEC e do procedimento cirúrgico.

Satyer e cols. ${ }^{9}$, em estudo com 106 crianças menores de 1 ano, concluíram igualmente que, naquelas com hiperfluxo pulmonar, a correção cirúrgica melhora a função pulmonar. Apesar da semelhança entre os resultados, tal estudo mede a Cp dinâmica, enquanto nós obtivemos a medida da Cst.

Lanteri e cols. ${ }^{10}$, em estudo com 23 crianças submetidas à cirurgia cardíaca, com idades entre 2 meses e 10 anos, similar aos nossos achados, observaram anormalidades da complacência dinâmica e estática nos pulmões e aumento da resistência respiratória entre os pacientes com hiperfluxo sangüíneo pulmonar. Essas medidas apresentaram valores normais depois da operação.

Takeuchi e cols. ${ }^{11}$ incluíram em seu estudo apenas crianças com peso inferior a $10 \mathrm{~kg}$ e também observaram melhora da Cp após a operação de correção do hiperfluxo pulmonar.

A análise do IC com as variáveis do estudo mostrou que apenas a idade, o peso e o tempo de perfusão apresentaram algum padrão de relação com IC.

Os pacientes com idade de até 30 meses apresentaram valor de IC, nos quatro instantes, mais concentrados e baixos. Quando a idade se eleva acima dos 30 meses, a dispersão dos valores de IC aumenta.

No subgrupo de pacientes com idade menor ou igual a 30 meses, o aumento percentual do IC é de apenas $4,1 \%$ ( $p=$ $0,0165)$, enquanto, no subgrupo de pacientes maiores de 30 meses, esse aumento é de $12,1 \%(p=0,0004)$.

$\mathrm{O}$ fato de as crianças menores de 30 meses apresentarem Cp menor em todos os instantes quando comparadas àquelas maiores de 30 meses e de o peso se comportar de forma semelhante nas crianças com menos de $10 \mathrm{~kg}$, apresentando, em todos os instantes, médias de Cp menor, deve-se provavelmente à maturação pulmonar.

Considerando-se a tabela de crescimento adotada mundialmente pela National Center of Health Statistics (NCHS), podemos observar que crianças abaixo de 30 meses são aquelas com peso até aproximadamente $10 \mathrm{~kg}$. Dessa forma, nossos resultados obtidos com as variáveis peso e idade foram compatíveis e mostraram-se similares para crianças de menor peso e menor idade, e também para aquelas de maior peso e maior idade.

Estudos sobre maturação pulmonar sugerem que o número de alvéolos aumenta até aproximadamente 8 anos de idade. Ao nascimento, a região alveolar é representada por espaços aéreos primitivos descritos como sáculos, chegando a quase vinte milhões. A partir daí, os alvéolos aparecem e se multiplicam para o número do adulto de trezentos milhões. A seguir, ocorrerá apenas o aumento de tamanho alveolar até a idade adulta ${ }^{2}$, o que contribui para uma Cp maior apenas a partir da idade aproximada dos 8 anos de vida. Outros autores que estudaram a maturação pulmonar mostram que, após os 4 meses de idade, aumenta a complexidade do formato alveolar, ocorrendo a multiplicação mais rápida dos alvéolos nos primeiros 3 anos de vida ${ }^{12,13}$. Isso certamente contribui para que a Cp seja maior quanto maior for a idade.
As crianças maiores apresentaram melhora da Cp do instante 1 para o instante 4 de $12 \%$. As crianças menores de 30 meses e com menos de $10 \mathrm{~kg}$ apresentaram também melhora da complacência ao final da cirurgia, mas de apenas 4,1\%.

Dessa forma, a Cp ao final da operação apresenta melhora significativa no grupo de crianças maiores. As menores mostraram menor variação do IC em todos os instantes, ficando esses valores concentrados num menor intervalo de variação, enquanto, naquelas maiores, os valores de IC mostraram maior variação.

Podemos, assim, inferir com mais precisão que, no grupo de crianças menores, a complacência estática pulmonar será menor em todos os instantes se comparada com crianças maiores de 30 meses.

É importante salientar que o aumento da Cp no pósoperatório imediato não apresentou nenhum padrão de relacionamento com o grau de hiperfluxo pulmonar, isto é, quando relacionamos apenas o $\mathrm{Qp} / \mathrm{Qs}$ com o grau de melhora da função pulmonar no pós-operatório, não observamos correlação positiva.

A não-relação do Qp/Qs com a evolução pulmonar indica a não-existência de interferência do grau de fluxo pulmonar com a capacidade pulmonar em pacientes com hiperfluxo pulmonar.

Alguns autores sugerem também que apenas a presença de hiperfluxo pulmonar com aumento da PAP (PAP $>18$ $\mathrm{mmHg}$ ) estaria associada à diminuição da complacência após a correção cirúrgica do hiperfluxo, isto é, a melhora é inversamente proporcional à PAP existente no pré-operatório. Quanto maior a PAP no pré-operatório, menor a melhora da $\mathrm{Cp}^{11}$.

Quanto à permanência em circulação extracorpórea, no grupo de crianças com tempo de perfusão menor ou igual a 50 minutos e também no grupo de crianças com tempo de perfusão maior que 50 minutos, houve melhora da Cp. Entretanto, no grupo que permaneceu menos tempo em CEC, já se observava a melhora da Cp desde o instante 3, quando o tórax ainda se encontrava aberto e os afastadores estavam posicionados. Já no grupo que ficou mais tempo em CEC, a melhora da Cp só foi observada ao final da operação, com o tórax fechado. Estes últimos mostraram inclusive piora do IC após a saída de CEC (instante 3).

O tempo de CEC é citado na literatura como um dos principais fatores que dificultam o desmame da VM em pacientes submetidos à cirurgia cardíaca, em razão do importante distúrbio fisiológico causado pela reação inflamatória. A alteração na membrana dos capilares pulmonares influencia negativamente nas trocas gasosas, com aumento do risco cirúrgico ${ }^{14}$.

Brown e cols. ${ }^{14}$, em determinado estudo para identificar fatores de risco para maior tempo em UTI, no pós-operatório de cirurgia cardíaca pediátrica, identificaram o tempo de CEC maior de 30 minutos como fator determinante para a piora da mecânica pulmonar e, conseqüente, aumento do tempo de UTI, independentemente dos eventos pósoperatórios subseqüentes.

Diversos efeitos adversos estão associados ao uso da 
CEC em crianças. O aumento da permeabilidade capilar leva à formação de edema, com conseqüente redução da complacência pulmonar e das trocas gasosas.

Os esforços para reduzir os efeitos adversos da CEC e deletérios da síndrome do extravasamento capilar pós-perfusão incluem, no intra-operatório, além de redução de volume dos circuitos e terapia diurética e antiinflamatória, a técnica de ultrafiltração. Estudos relacionados à hemoconcentração em cirurgias cardíacas com CEC têm sido feitos e concluem que as principais vantagens incluem a remoção de macromoléculas mediadoras da reação inflamatória sistêmica, diminuição de endotoxinas circulantes, remoção do excesso de água corpórea e da hipertensão pulmonar ${ }^{15,16}$.

Verificou-se um dado interessante na correlação entre o tempo de CEC e a idade. Quando analisamos os dados intergrupos, as crianças com menor tempo de CEC apresentavam valores significativamente maiores de Cp em todos os instantes medidos, quando comparadas àquelas do grupo com maior tempo de CEC. Observou-se que as primeiras tinham uma média de idade maior que as últimas. As crianças maiores ficaram menos tempo em CEC que as menores. A justificativa para tal evento pode ser encontrada no fato de a indicação da operação mais precoce acontecer na presença de defeitos mais complexos, que causam maior repercussão hemodinâmica, o que explica o maior tempo de CEC.

$A R_{V A}$ no presente estudo não apresentou alteração significativa ao final do procedimento cirúrgico $(p=0,393)$. Di Carlo e cols. ${ }^{17}$ relacionaram o valor medido de $R_{V A}$ no pós-operatório imediato com o sucesso ou insucesso na extubação e constataram que valores de $R_{V A}$ superiores a 75 $\mathrm{cm} \mathrm{H}_{2} \mathrm{O}$ indicam a possibilidade de insucesso na extubação por insuficiência respiratória. Referiram também que o aumento da $R_{V A}$ se deve provavelmente ao acúmulo de líquido pulmonar. Esse estudo foi realizado com crianças submetidas a diversos tipos de cirurgias cardíacas, e não somente para correção de hiperfluxo, como é o caso do presente estudo ${ }^{17}$.

Nosso estudo não encontrou relação da alteração da $R_{v A}$ com nenhuma variável do pré, intra ou pós-operatório. Dessa forma, não houve relação da $\mathrm{R}_{\mathrm{VA}}$ com o tempo de $\mathrm{VM}$, embora a média da $R_{V A}$ ao final da cirurgia tenha ficado abaixo de $75 \mathrm{~cm} \mathrm{H}_{2} \mathrm{O}$.

\section{Referências}

1. Lister G, Pitt BR. Cardiopulmonary interactions in the infant with congenital cardiac disease. Clin Chest Med. 1983; 4 (2): 219-32.

2. Reid LM. Lung growth in health and disease. Br J Dis Chest. 1984; 78: 113-24.

3. Babik B, Astalos T, Peták F, Deák ZI, Hantos Z. Changes in respiratory mechanics during cardiac surgery. Anesth Analg. 2003; 96: 1280-7.

4. Vieira SRR, Plotnik R, Fialkow L. Monitorização da mecânica respiratória durante a ventilação mecânica. In: Carvalho CRR (ed). Ventilação mecânica. São Paulo: Atheneu; 2000. p. 215-52.

5. Carvalho AP, Ayres FC. Circulação e respiração: fundamentos da biofísica e fisiologia. 3a ed. Rio de Janeiro: Cultura Médica; 1983. p.175.
$A R_{V A}$ é maior nos menores volumes pulmonares. Tal situação parece estar relacionada ao fato de ocorrerem alterações nos diâmetros das vias aéreas com diferentes volumes pulmonares ${ }^{4}$. Isso explica o que nossos resultados demonstraram, ou seja, que as maiores resistências foram encontradas no grupo de crianças menores de 30 meses e que pesavam menos que $10 \mathrm{~kg}$.

As variações da $R_{v A}$ são muito pequenas nos dois grupos ao longo do período intra-operatório, não sendo significativas as alterações ao final da cirurgia.

Contrariamente, estudos mostram que pacientes neonatais tiveram redução na resistência respiratória de 25\% após a cirurgia, enquanto crianças mais velhas apresentaram redução de apenas $16 \%{ }^{9}$.

Segundo Martins ${ }^{18}$, a monitorização de diferentes aspectos da função respiratória pode não só prevenir complicações, como também permitir melhor avaliação funcional do paciente. Assim, a medida de Cst não-invasiva do sistema respiratório deve ser utilizada rotineiramente na prática clínica e principalmente no pós-operatório das cirurgias cardíacas. É adequada para avaliação do parênquima pulmonar, pois os sistemas cardiovascular e respiratório estão intimamente relacionados, tanto anatômica quanto funcionalmente.

Em nosso estudo, os principais achados foram: as crianças apresentaram melhora do IC após a correção cirúrgica do hiperfluxo pulmonar; as crianças de menor idade e, portanto, de menor peso apresentaram essa melhora de forma mais discreta; aquelas que permaneceram mais tempo em CEC demoraram mais tempo para apresentar melhora do IC. O IR não mostrou alteração significativa ao final da cirurgia.

\section{Potencial Conflito de Interesses}

Declaro não haver conflito de interesses pertinentes.

\section{Fontes de Financiamento}

O presente estudo não teve fontes de financiamento externas.

\section{Vinculação Acadêmica}

Este artigo é parte de tese de mestrado de Lilian Goraieb pela Faculdade de Medicina de São José do Rio Preto.

6. Barnas GM, Watson RJ, Green MD, Sequeira AJ, Gilbert TB, Kent J, et al. Lung and chest wall mechanical properties before and after cardiac surgery with cardiopulmonary bypass. J Appl Physiol. 1994; 76: 166-75.

7. Zin WA, Rocco PRM. Mecânica respiratória nomal. In: Auler Junior JOC, Gomide do Amaral RV (eds). Assistência ventilatória mecânica. São Paulo: Atheneu; 1995. p. 3-24.

8. Schulze-Neick I, Li J, Penny DJ, Redington AN. Pulmonary vascular resistance after cardiopulmonary bypass in infants: effect on postoperative recovery. J Thorac Cardiovasc Surg. 2001; 121: 1033-9.

9. Stayer SA, Diaz LK, East DL, Gouvion JN, Vencill TL, McKenzie ED, et al. Changes in respiratory mechanics among infants undergoing heart surgery. 
Anesth Analg. 2004; 98: 49-55.

10. Lanteri CJ, Kano S, Duncan AW, Sly PD. Changes in respiratory mechanics in children undergoing cardiopulmonary bypass. Am J Respir Crit Care Med. 1995; 152 (Pt 1): 1893-900

11. Takeuchi M, Kinouchi K, Fukumitsu K, Kishimoto H, Kitamura S. Postbypass pulmonary artery pressure influences respiratory system compliance after ventricular septal defect closure. Pediatr Anaesth. 2000; 10: 407-11.

12. Davies G, Reid L. Growth of the alveoli and pulmonary arteries in childhood. Thorax. 1970; 25: 669-81.

13. Hislop A, Reid LM. Pulmonary arterial development during childhood: branching pattern and structure. Thorax. 1973; 28: 129-35.

14. Brown KL, Ridout DA, Goldman AP, Hoskote A, Penny DJ. Risk factors for long intensive care unit stay after cardiopulmonary bypass in children. Crit Care Med. 2002; 31: 1

15. Souza DD, Braile DM. Assessment of a new technique of hemoconcentration and the necessities of blood derivates for transfusion in patients submitted to heart surgery using cardiopulmonay bypass. Braz J Cardiovasc Surg. 2004; 19 (3): 287-94.

16. Elliot MJ. Ultrafiltration and modified ultrafiltration in pediatric open heart operations. Ann Thorac Surg. 1993; 56 (6): 1518-22.

17. Di Carlo JV, Raphaely RC, Steven JM, Norwood WI, Costarino AT. Pulmonary mechanics in infants after cardiac surgery. Crit Care Med. 1992; 20 (1): 22-7.

18. Martins F. Monitorização no pós-operatório de cirurgia cardíaca. In: Auler Jr JOC, Oliveira AS (eds). Pós-operatório de cirurgia torácica e cardiovascular. Porto Alegre: Artmed; 2004. p.18-42. 